\title{
Spectroscopic Studies of Nano Size Crystalline Conducting Polyaniline
}

\author{
B. Kavitha ${ }^{1}$, K. Prabakar $^{2}$, K. Siva kumar ${ }^{3}$, D. Srinivasu ${ }^{4}$, \\ Ch. Srinivas ${ }^{5}$, V.K.Aswal ${ }^{6}$, V. Siriguri ${ }^{7}$, N.Narsimlu ${ }^{8}$ \\ 1, 2, 3, 4, 5, 8 Department of Physics, Osmania University, Hyderabad - 500004 INDIA \\ ${ }^{6}$ SSP Division (BARC), ${ }^{7}$ UGC-DAE CSR, Bhaba Atomic Research Center (BARC) Mumbai, INDIA 400085
}

\begin{abstract}
Polyaniline (PANI) was synthesized in Emaraldine form by in situ chemical oxidation method by using Ammonium perdisulphate as oxidizing agent. The XRD pattern indicates that PANI is a semi crystalline solid with d-spacing $4.801 \dot{A}$ and $4.358 \not A$. The SEM results show that the particle size lies as an average of 80 $\mathrm{nm}$ and length 550nm. Similarly the UV-Visible spectra of this polymer indicate two absorption bands at around $320 \mathrm{~nm}$ and 640nm. These absorption bands are attributed to the transitions of $\pi \rightarrow \pi^{*}$ and Benzenoid to Quinoid respectively. The FT-IR spectrum of PANI shows strong bands at 3442, 2925, 1598, 1494, 1453, 1176, 1112 and $744 \mathrm{~cm}^{-1}$ respectively. ${ }^{1} H$ NMR spectrum shows 6 peaks with chemical shifts $\delta=7.452,7.468,7.505$, $7.525,7.604,7.638 \mathrm{ppm}$. The lower three peaks at $(\delta=7.452,7.468,7.505 \mathrm{ppm})$ are attributed to the protons related to the ${ }^{14} N$ nucleus. These spectral lines intensity ratio lies as 1:1:1. Another three peaks at $(\delta=7.525$, 7.604, 7.638 ppm) are attributed to the three protons, which are present at CH Benzenoid, NH, CH at Quinoid. The intensity ratio of these spectral lines is 1:2:1. The room temperature ESR spectrum of PANI Emeraldine salt shows an unresolved peak with lande's $g$ factor 2.010 due to polaron.
\end{abstract}

Key words: Polyaniline (PANI), XRD, FTIR, NMR, ESR.

\section{Introduction}

Over 30 years ago conjugated polymers were as futuristic new materials that would lead to the next generation of electronic and optical devices. The development of "plastic electronics" devices based on conjugated polymers has evolved the states of these materials from academic curiosity to the rapidly growing new electronic industry. Among these conjugated polymers polyaniline possess excellent thermal and environmental stability. Polyaniline have been used in variety applications like diodes, Field effect transistors, solar cells, memory devices, corrosion resistance and in gas sensors [1]. The polyaniline can be synthesized in to different sizes and shapes like nanofibers, nanorods, nanotubes and in to thin films. Not only tunable morphology, polyaniline can be synthesized with tunable properties like electrical conductivity and optical band gap. The presence of Imines and amine nitrogen functional groups in the polyaniline is the main reason for the tunable properties of polyaniline [Fig. 1] These two peculiar properties have given a lot of importance to polyaniline in nano-electronic and optical devices technology. A morphological and spectroscopic study of these polymers is an important subject to understand the transport phenomena of these conjugated polymers to apply them in electronic industry [2,3]. Hence we attempted to synthesis the polyaniline nanofibers, and characterization of these synthesized nano-polymers with help of different spectroscopic techniques.

\section{Synthesis of Polyaniline:}

\section{Experimental Section}

Polyaniline was synthesized by In-Situ chemical polymerization of aniline in the presence of hydrochloric acid as a catalyst and ammonium peroxydisulphate as an oxidant. For the synthesis, we took 50ml, $1 \mathrm{M} \mathrm{HCl}$, and $2 \mathrm{ml}$ of aniline were added into a $250 \mathrm{ml}$ beaker equipped with a Teflon coated magnetic stirrer at about $0^{\circ} \mathrm{C}$ temperature. Then $5 \mathrm{~g}$ of ammoniumperdisulphate $\left(\left(\mathrm{NH}_{4}\right)_{2} \mathrm{~S}_{2} \mathrm{O}_{8}\right)$ aqueous solution in $50 \mathrm{ml} 1 \mathrm{M} \mathrm{HCl}$ was drop wise added into the above solution. The polymerization temperature $0^{\circ} \mathrm{C}$ was maintained for $10 \mathrm{~h}$ to complete the reaction. Then the precipitate obtained was filtered. The product was washed successively by $1 \mathrm{M}$ $\mathrm{HCl}$ followed by double distilled water until the wash solution turned colorless. The product PANI was dried at $60^{\circ} \mathrm{C}$ for $24 \mathrm{~h}$ to get powder form PANI. This powder was pelletized with help of hydraulic machine for characterization.

\section{Characterization:}

The X-Ray diffraction studies of polyaniline were performed on Phillips XPERT diffractometer with $\mathrm{Cu} \mathrm{K}_{\alpha} \mathrm{X}$-ray $(\lambda=1.54 \AA$ ). Scanning electron microscope (SEM) experiments are performed by employing Hitachi SU 1510 microscope. The Fourier transform infrared (FTIR) spectra were obtained using a Bio-rad FTIR (model FT-155) spectrophotometer. The samples were mixed with $\mathrm{KBr}$ and pressed in to a pellet. The room 
temperature optical absorption spectrum of these materials was recorded by employing the Varian spectrometer (model Cary 5E) in the 190nm to $1000 \mathrm{~nm}$ wavelength region. The ESR spectra were recorded by using JEOL spectrometer and NMR studies are performed in CFRD Osmania University by employing the Bruker spectrometer.

\section{Results And Discussion}

The X-ray diffraction pattern of polyaniline emeraldine salt form shows two sharp peaks at $2 \theta=21.10$ and 23.59. The inter-planar distances are calculated as $42.10 \mathrm{~nm}$ and $37.70 \mathrm{~nm}$ respectively. The average crystallite size is calculated by Debye Scherer equation $D=[0.89 \lambda] /[\beta \operatorname{Cos} \theta]$ respectively, where $\beta$ is full width of half maxima (FWHM) [Fig.2]. Generally polymers are considered to be amorphous, but here the synthesized polymer is showing crystalline structure due to their fiber nature and planar nature of Benzenoid and Quinoid functional groups [3]. Similarly polyaniline emeraldine base form shows only one peak at $19^{0}$. The SEM micrographs reveal that they are actually agglomeration of nanofibers [Fig.3]. The diameter and length of nanofibers are measured as $80 \mathrm{~nm}$ and $550 \mathrm{~nm}$ respectively. Agglomeration is taking place due to the conventional filtration of polymer powder in the synthesis process. The Fourier transform infrared spectra [Fig. 4.1] of Polyaniline nanofibers are also consistent with the existing literature with strong bands at around 1558 and $14690 \mathrm{~cm}^{-1}$ corresponds to Benzenoid and Quinoid rings and the remaining bands assigned to their respective functional groups [5] as shown in Table 1.

The UV-Visible spectrum of Polyaniline Emeraldine base form shows two absorption peaks at 325 and $625 \mathrm{~nm}$ (Fig.5). These peaks are assigned to the $\pi-\pi^{*}$ transition and polarans band transitions respectively. Similarly, the UV-Visible spectrum of HCl doped Polyaniline emeraldine salt shows three absorption bands at $340 \mathrm{~nm}, 440 \mathrm{~nm}$ and $800 \mathrm{~nm}$ (Fig.6). They are the $\pi-\pi^{*}$ transition located $340 \mathrm{~nm}$, the polaron- $\pi^{*}$ transition at $440 \mathrm{~nm}$ and polaron- $\pi$ transition around $800 \mathrm{~nm}$. This indicates that the synthesized polyaniline have been effectively doped protonic acid. These two features are fingerprint of electronically conducting Polyaniline Emeraldine salt form $[6,7]$.

Table .1 FTIR peak assignment of polyaniline emeraldine form

\begin{tabular}{|c|c|c|}
\hline S.No. & Peak position $\mathrm{cm}^{-1}$ & Assignment \\
\hline 1 & 3442 & O-H Stretching \\
\hline 2 & 2925 & N-H Bending \\
\hline 3 & 1598 & Quinoid deformation \\
\hline 4 & 1494 & Benzenoid deformation \\
\hline 5 & 1112 & C-N=C stretching \\
\hline 6 & 744 & C-H out of plane bending \\
\hline
\end{tabular}

The room temperature ${ }^{1} \mathrm{H}$ NMR spectrum of polyaniline shows 6 peaks with chemical shifts $\delta=7.452$, $7.468,7.505,7.525,7.604,7.638 \mathrm{ppm}$ (Fig.7). The lower three peaks at $(\delta=7.452,7.468,7.505 \mathrm{ppm}$ ) are attributed to the protons related to the ${ }^{14} \mathrm{~N}$ nucleus. These spectral lines intensity ratio lies as 1:1:1. Another three peaks at $(\delta=7.525,7.604,7.638 \mathrm{ppm})$ are attributed to the three protons, which are present at $\mathrm{CH}$ Benzenoid, NH, CH at Quinoid [8-10]. The intensity ratio of these spectral lines is 1:2:1. The ESR spectrum of PANI Emeraldine salt form at room temperature shows an unresolved peak with lande's g factor 2.010 ( $h v=$ $\mathrm{g} \beta \mathrm{H})$. This may be attributed to the half spin polarans which is created in the PANI back bone due to doping of $\mathrm{HCl}$ acid. This result is quite supporting the UV-Visible spectra of PANI Emeraldine salt form. As polyaniline is doped, the resonance line amplitude of the positive lobe (I+) is not equal to the amplitude of the negative lobe, (I-). This change in the resonance line shape is due to the skin effect in polyaniline [11, 12].

\section{Conclusion}

We synthesized polyaniline nano-fibers by chemical oxidative polymerization method. The morphological studies (XRD, SEM) indicate the radius of polymer fiber is around 80nm and length around 550 $\mathrm{nm}$ respectively. The Emeraldine salt form of polyaniline is confirmed with FTIR, UV/Visible and ESR spectra. The proton NMR studies reveals that peak at a triplet is caused by -NH free radical. ESR studies reveal the formation of polarans in polyaniline emeraldine salt form.

\section{Acknowledgements}

The Authors ${ }^{1}$ are thankful to the UGC-DAE CSR Mumbai center for sanctioning the research (CRS) project No. UDCSR/MUM/AO/CRS-M-181/12/035. 
A.J. Heegar Nobel lecture December 8 (2000)

\section{References}

[2] Cheng-Ho Chen. Journal of Applied Polymer science.89, 2142-2148 (2003)

[3] Jianyong Ouyang, Chih- Wei Chu, Ricky J.Seng, Ankitha Prakash and yang Yang. Hand Book of conducting polymers. Proceesing and applications.8.-1Third Edition (2006)

[4] S.K.Shukla, A. Bhradvaja, A.Tiwari, Srikant pilla, G.K. Parashar and G.C.Dubey. Adnance materials letters1(2) 129-134 (2010)

[5] Ricardo H.Goncalves, Wado Herving Schreiner and Edson R. Leite. Langmuir Articl.26(14) 11657-11662.( 2010)

[6] Jiaxing Huang and Richard B. Kaner Handbook of Conducting Polymers Third Edition Conjugated Polymers

Edited by Terje A. Skotheim and John R. Reynolds Conjugated Polymers: Theory, Synthesis, Properties, and Characterization CRC

Press Taylor \& Francis Group. 7-16

[7] J.Huang,and Richard B.Kaner Hand Book of Conjugated polymers, third edition 7-14 (1998)

[8] ShaolinMu and Yifel Yang. J.Phys. Chem. B.112, 11558-11563 (2008)

[9] K.Gupta, P.C.Jana and A.K. Melkap Journal of Physicsl Sciences. 12, 233-238. (2008)

[10] D.B.Dupare, M.D.Shirsat, A.S.Aswar Yhe Pacific Journal of Science and Technology. 10, 1, may (2009)

[11]. D. Stauffer, A. Aharony, Introduction to percolation theory, Taylor \& Francis, London, 1992.

[12]. M. Chipara,Gh. Aldicaa, D. Huib, M. Dimoniec, K. T. Laud, L. Georgescue, I. Munteanue, H. Marascoiuc Journal of Optoelectronics and Advanced MaterialsVol. 6, No. 1, March 2004, p. 297 - 305

Figure Captions:

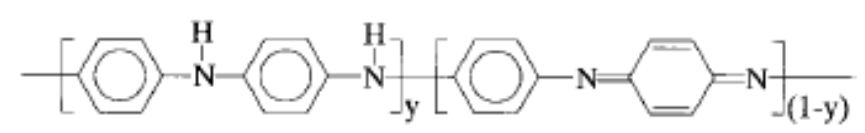

Fig.1. Polyaniline Chemical Structure

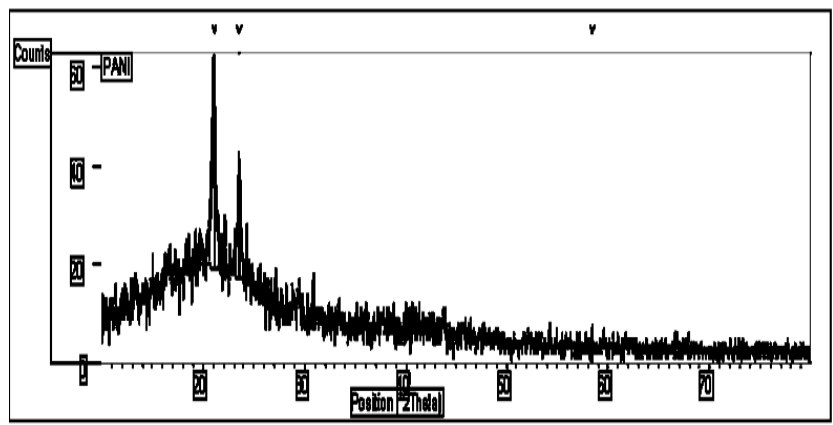

Fig.2 XRD Pattern of Polyaniline

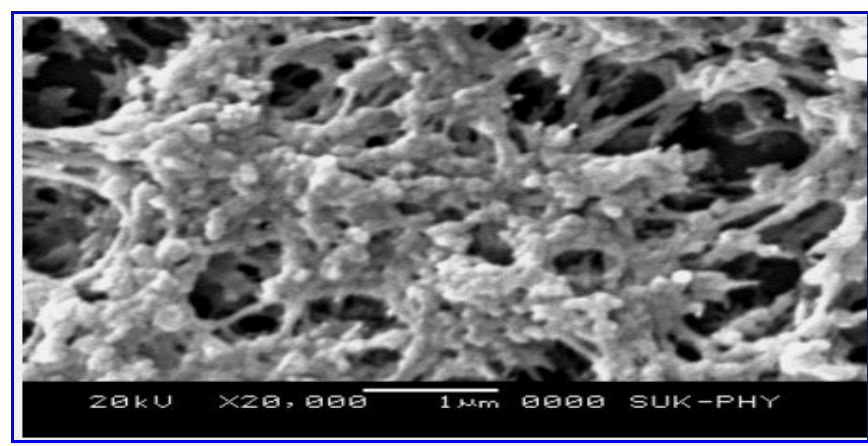

Fig.3 SEM image of Polyaniline

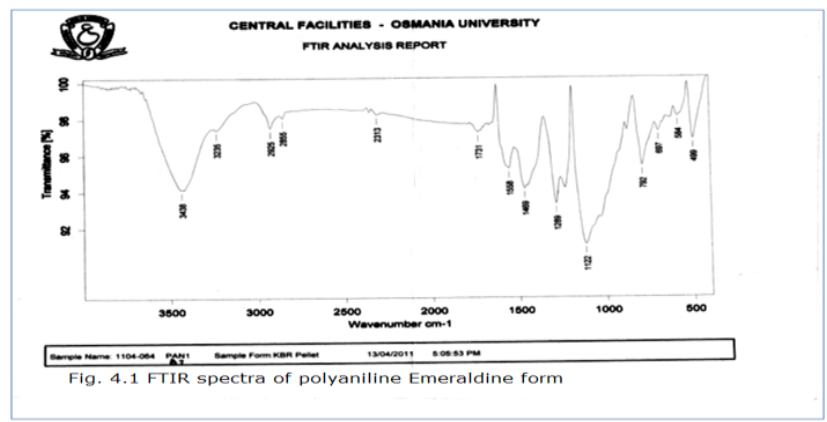




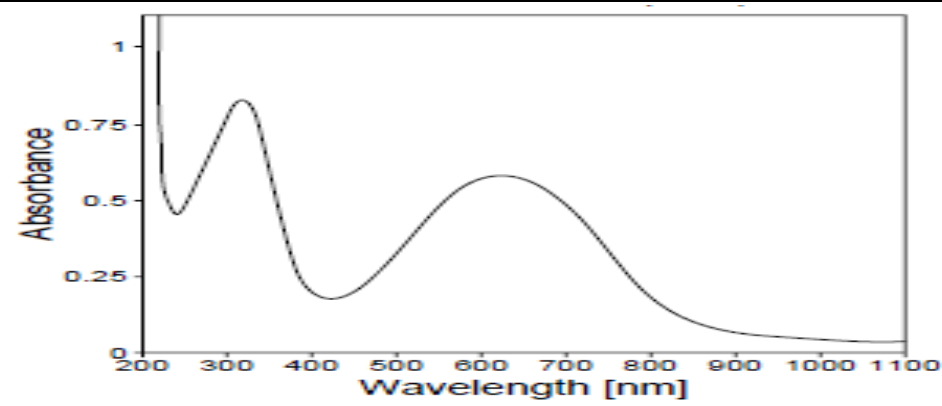

Fig.5 UV/Visible spectra of Polyaniline Emeraldine base form.

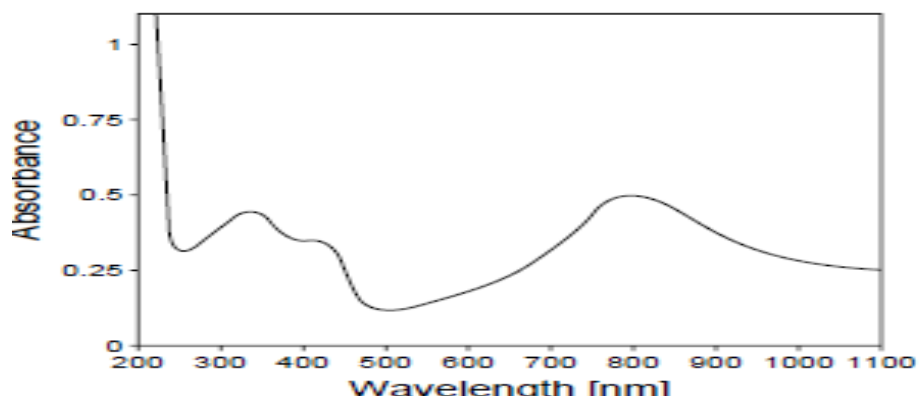

Fig.6 UV/Visible spectra of Polyaniline Emeraldine salt form.

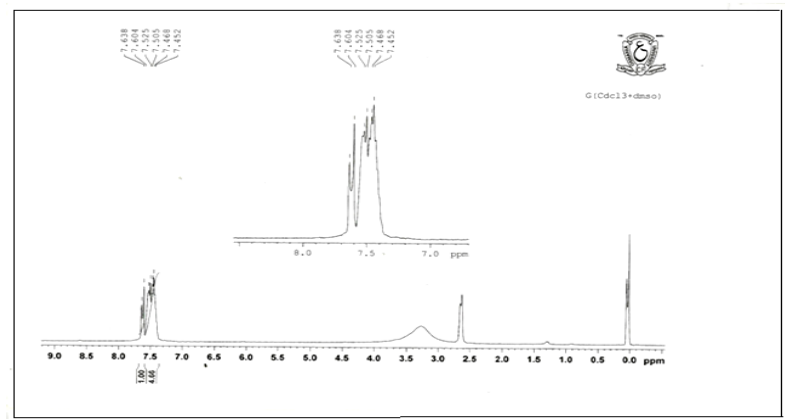

Fig. $7 \mathrm{H}^{1}$ NMR spectra of Polyaniline 